4

5

6

7

8

\title{
A model for estimating traction force magnitude reveals differential regulation of actomyosin activity and matrix adhesion number in response to smooth muscle cell spreading
}

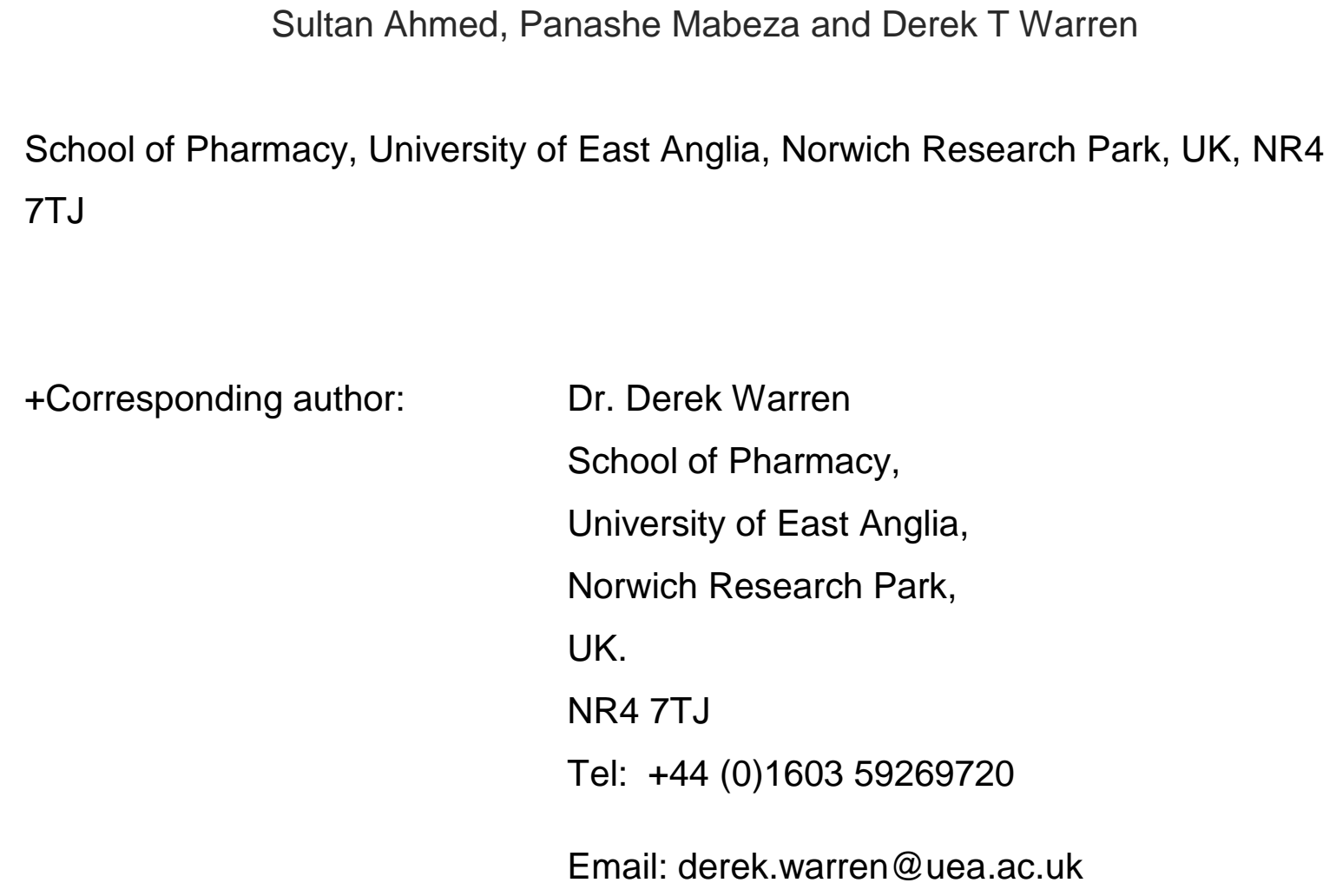

Email: derek.warren@uea.ac.uk

\section{Running Head: VSMC spreading predicts traction force}

Word count: 4,392 


\section{Abstract}

Decreased aortic compliance is associated with ageing and vascular disease, including atherosclerosis and hypertension. Ultimately, changes in aortic compliance are driven by altered ECM composition however, recent findings have identified a cellular component to decreased aortic compliance observed in ageing and hypertension. Vascular smooth muscle cells (VSMCs) line the blood vessel wall and VSMC contraction regulates vascular tone and contributes to aortic compliance. Mechanical cues derived from the ECM influence VSMC function, yet whether ECM rigidity influences VSMC force generation remains unclear. In this study, we describe the relationship between VSMC spreading, traction force magnitude and matrix rigidity. Importantly, we show that spreading predicts integrated traction force (integrated-TF) magnitude independently of matrix rigidity. Using linear regression analysis, we have generated a model for calculating integrated-TF from VSMC area. This model closely predicts the integrated traction force measured by live VSMC traction force microscopy. Vinculin staining analysis revealed that spreading strongly correlated with adhesion number per VSMC, suggesting that increased VSMC integrated-TF was due to enhanced matrix anchor points. Further analysis revealed that calculated integrated-TF per adhesion was reduced by matrix rigidity, however, adhesion number $/ \mu \mathrm{m}^{2}$ increased, resulting in the average integrated-TF/ $\mu \mathrm{m}^{2}$ remaining unaltered. As a result, the integrated-TF/VSMC spreading relationship is independent of matrix rigidity. Therefore, our study has identified and validated a novel model to predict and understand the mechanisms influencing VSMC traction force magnitude.

Key Words: Vascular smooth muscle cells, traction force, matrix rigidity

\section{1}




\section{Introduction}

Decreased vascular compliance is a risk factor in the development of cardiovascular disease, including atherosclerosis and hypertension $(1,2)$. Rodent models of hypertension display a 4-fold increase in aortic stiffness compared to age matched controls (3). Additionally, the Young's modulus, E, (a measure of material stiffness) of healthy aorta has been determined by atomic force microscopy to be between $10-20 \mathrm{kPa}$, whereas atherosclerotic plaques contain a stiffened fibrous cap $(E=60-250 \mathrm{kPa})$ (4). Ultimately, these changes in aortic compliance are driven by altered extracellular matrix (ECM) composition. Elastic ECM components, including elastin, are degraded and non-elastic ECM components, such as collagen-1, accumulate $(5,6)$. However, recent studies have identified a cellular component to aortic compliance $(3,7-9)$. Vascular smooth muscle cells (VSMCs) are the predominant cell type in the medial layer of the aortic wall and normally exist in a quiescent, contractile phenotype where actomyosin-derived contractile forces maintain vascular tone (7). However, VSMCs display remarkable plasticity and during vessel remodelling, they undergo phenotypic transition to a synthetic, migratory phenotype. This synthetic phenotype is prevalent in vascular disease, including atherosclerosis and aneurysm $(10,11)$. Phenotypic modulation is associated with changes in VSMC contractile protein expression and morphology (12); however, we still do not fully understand whether the ability of VSMCs to generate actomyosin derived-force is altered by VSMC phenotypic modulation.

Microenvironment rigidity transmits 'outside-in' forces to VSMCs and this process is dependent on adhesions that convey force between the ECM and cytoskeleton (13-16). VSMCs and other cell types respond to outside-in signals by exerting actomyosin based contractile forces on the matrix (inside-out forces) that scale with ECM stiffness $(13,15,16)$. Rho/ROCK signalling is rapidly activated at ECM adhesions in response to matrix rigidity, to augment actomyosin activity, via actin polymerisation and myosin light chain phosphorylation (15). Actin cytoskeletal reorganisation and enhanced actomyosin activity increase VSMC integrated traction forces, the force VSMCs apply to the ECM. In other cell types, matrix rigidity promotes enhanced adhesion and actomyosin activity to increase traction force (17-20). However, our knowledge of the balance between actomyosin activity, adhesion organisation and traction force magnitude in response to matrix rigidity in VSMCs remains limited. Traction force microscopy (TFM) remains the gold standard for 
measuring traction forces generated by cells, but is highly specialised and time consuming to perform. As such, only a limited number of studies have measured VSMC traction force. Those that have been performed have reported that matrix rigidity promotes increased traction force magnitude and adhesion reorganisation (2123). However, the mechanisms driving these changes remain unknown. Therefore, in this current study we investigate the impact of matrix rigidity on VSMC traction force generation. We show that VSMC spreading and integrated traction force (integratedTF) magnitude display a moderate correlation and that this relationship was independent of matrix rigidity. Importantly, we show that actomyosin activity and adhesion number are inversely regulated by matrix rigidity and VSMC spreading. This inverse regulation results in a net zero change in TF/ $\mu \mathrm{m}^{2}$. However, VSMC spreading results in increased adhesive contractile units per cell which augments integrated-TF. Our study suggests that VSMC traction force is tightly regulated by a compensatory mechanism involving differential actomyosin and adhesion regulation.

\section{Methods}

\section{Cell culture}

Human adult aortic VSMCs (passage 3-10) were purchased from Cell Applications Inc (Isolate-1). Human adult aortic 35F VSMCs were a kind gift from Professor Cathy Shanahan (King's College London, UK) (Isolate-2). VSMCs were grown in growth media (Cell Applications Inc) and were otherwise cultured as described previously (24).

\section{Polyacrylamide hydrogel preparation and Traction Force Microscopy}

Hydrogels were prepared as described previously $(25,26)$. A JPK Nanowizard3 atomic force microscope was used to confirm hydrogel stiffness as described previously (25). VSMCs were seeded onto $12 \mathrm{kPa}$ hydrogels containing $0.5 \mu \mathrm{m}$ red fluorescent (580/605) FluoSpheres (Invitrogen). Imaging was performed using a Nikon Eclipse Ti-E live cell imaging system to capture 20x magnification images before and after cell lysis by the addition of $0.5 \% \mathrm{Tx}-100$. Drift was corrected using the ImageJ StackReg plugin and traction force was calculated using the ImageJ plugin described previously to measure FluoSphere displacement (27). Briefly, bead displacement was 
measured using the first and last image of the movie sequence. To determine integrated (cell total) the cell region was segmented by overlaying the traction map with the cell image, highlighting the cell traction region with an $\mathrm{ROI}$ and extracting the traction forces in each pixel by using the save $X Y$ coordinate function in ImageJ. To generate gradient trend line data, integrated-TF was plotted against VSMC area on a scatter plot in Graph Pad Prism 7. Linear regression analysis was performed to generate:

Formula 1: Calculated Integrated-TF $=3.007($ area $)-472.52$

Formula 2: Calculated Integrated-TF $=2.97($ area $)-66.913$

\section{Western blot analysis}

Antibodies used for WB, IF; Vinculin (V9131), $\alpha$-actin, $\beta$-actin (A5316) (Sigma). Secondary antibodies for WB were horseradish peroxidase-conjugated anti mouse (NA931) or anti rabbit (NA94V) antibodies from GE Healthcare. ECL chemiluminescent kit (RPN2132, GE Healthcare) was used for detection according to manufacturer's instructions.

\section{Fixed cell microscopy and data analysis}

Cells were cultured on hydrogels, fixed in paraformaldehyde and processed as described previously (28). Invitrogen anti-mouse Alexa fluor 568 (A11031) and antirabbit Alexa fluor 488 (A11034) were used as IF secondary antibodies. Rhodamine Phalloidin was used to stain F-actin and DAPI was used to visualise cell nuclei. All images were captured at 20x magnification using a Leica SP2 laser scanning confocal microscope. Cell area and aspect ratio was measured using the ImageJ open source analysis software (28). pMLC mean intensity and total intensity were measured using the ROI function in ImageJ. Focal adhesion number and size were measured in ImageJ, as described previously (28).

\section{Statistics}

Results are presented as mean +/- SEM. Results are presented to show data distribution and each point corresponds to any individual measurement. For comparison of matrix stiffness group's, paired student t-tests were performed. 
GraphPad Prism 7 software was used to generate trend-line gradient equations, scatter plots and to perform linear regression and statistical analysis.

\section{Results}

Matrix stiffness stimulates VSMC spreading and increases traction force magnitude

We set out to validate previous findings showing that matrix rigidity stimulates spreading and actomyosin activity in VSMCs. VSMCs were grown on collagen-1 coated polyacrylamide hydrogels, with an average Young's modulus of 12 (healthy) and $72 \mathrm{kPa}$ (stiff) (Supplementary Figure 1A). Western blot analysis revealed that levels of smooth muscle actin (SM-actin), calponin and $\beta$-actin remained unchanged by matrix stiffness (Supplementary Figure 1B), suggesting that matrix rigidity does not alter expression of VSMC contractile proteins. Immunofluorescence microscopy (IF) analysis confirmed that VSMCs were larger on $72 \mathrm{kPa}$ hydrogels compared to those grown on 12kPa hydrogels (Supplementary Figures $1 \mathrm{C}$ and $\mathrm{D}$ ). Aspect ratio remained unchanged by matrix stiffness (Figures $1 \mathrm{C}$ and $\mathrm{E}$ ). To observe cellular force generation, we performed traction force microscopy on VSMCs grown on 12 and $72 \mathrm{kPa}$ hydrogels. The ability of VSMCs to displace beads embedded in the hydrogels was reduced on $72 \mathrm{kPa}$ compared to $12 \mathrm{kPa}$ hydrogels (Figures $1 \mathrm{~A}$ and $\mathrm{B}$ ). However, integrated VSMC traction force (integrated-TF) magnitude was enhanced on the $72 \mathrm{Kpa}$ hydrogels compared to $12 \mathrm{kPa}$ hydrogels (Figures $1 \mathrm{~A}$ and $\mathrm{C}$ ). These data confirm previous findings showing that matrix rigidity influences VSMC spreading and traction force. 
A.

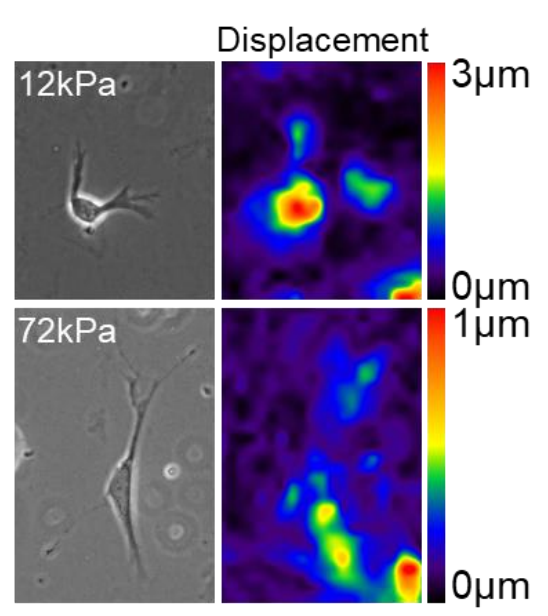

E.

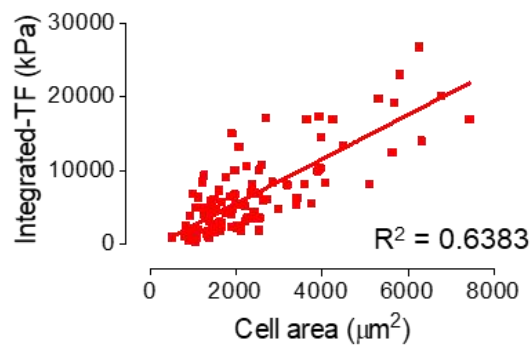

B.

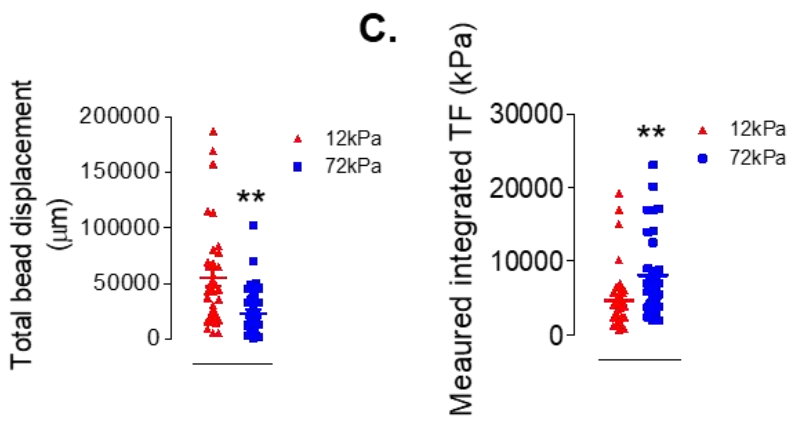

D.

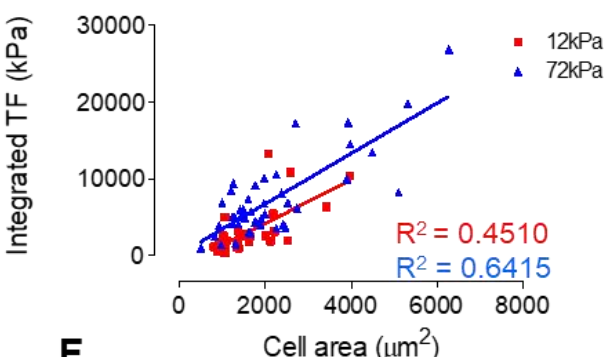

F.

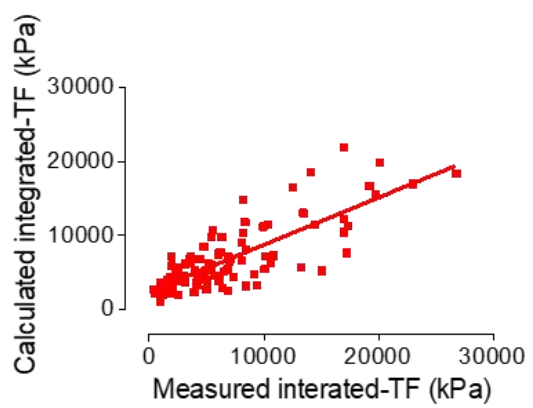

G.

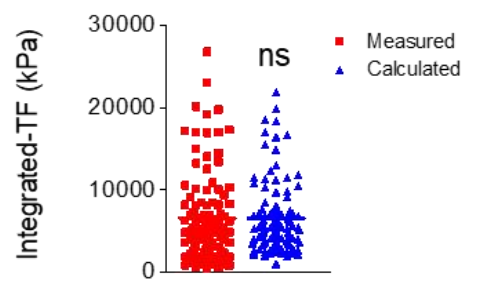

Figure 1. VSMC spreading/integrated-TF magnitude relationship is matrix rigidity independent. A) Representative bead displacement maps for isolate-1 VSMCs grown on 12 and $72 \mathrm{kPa}$ hydrogels. Graphs show B) integrated bead displacements and C) integrated-TF magnitude for VSMCs grown on $12(n=29)$ and $72 \mathrm{kPa}(n=42)$ hydrogels. $D)$ Graph shows distribution of integrated-TF magnitude plotted against VSMC area on $12 \mathrm{kPa}(\mathrm{red})(\mathrm{n}=29)$ and $72 \mathrm{kPa}$ (blue) $(n=42)$ hydrogels. E) Graph shows combined distribution of integrated-TF plotted against VSMC area on $12 \mathrm{kPa}$ and $72 \mathrm{kPa}$ hydrogels $(\mathrm{n}=104)$. Graphs show $\mathrm{F})$ distribution of calculated versus measured integratedTF magnitude and G) comparison of measured and calculated integrated-TF using the trend-line formula (formula 1) generated in E). (** $p=<0.01$ and $n s=$ non-significant). 
The above data shows that matrix rigidity enhances both VSMC spreading and integrated traction force magnitude. VSMC spreading has previously been reported to correlate with traction force magnitude on soft $1 \mathrm{kPa}$ hydrogels (29). We next speculated that matrix rigidity influences the relationship between VSMC spreading and traction force magnitude. Analysis revealed that a moderate correlation between VSMC spreading and integrated-TF magnitude existed on both $12 \mathrm{kPa}\left(\mathrm{R}^{2}=0.4510\right)$ and $72 \mathrm{kPa}\left(\mathrm{R}^{2}=0.6415\right)$ hydrogels (Figure 1D). Importantly, the relationship between VSMC spreading and integrated-TF was not significantly altered by matrix rigidity, suggesting that changes in traction force magnitude were driven by changes in VSMC spreading.

As the VSMC spreading/integrated-TF magnitude relationship was independent of matrix rigidity, we hypothesised that integrated-TF could be calculated from VSMC area. To test this idea, we combined the data from the $12 \mathrm{kPa}$ and $72 \mathrm{kPa}$ hydrogels and calculated the gradient of the trend line (Figure 1E). The formula generated was used to calculate integrated-TF magnitude from the measured VSMC area. As expected, the spread of calculated data closely resembled that of measured integrated-TF magnitude (Figures $1 F$ and $G$ ). To test this hypothesis further, we used an independent aortic VSMC isolate. TFM was performed to experimentally measure VSMC traction force. The measured data confirmed that bead displacement was reduced whereas integrated-TF magnitude was enhanced by matrix rigidity, recapitulating the above findings (Figures $2 A-C$ ). Similar to our above findings, the integrated-TF/spreading relationship for isolate-2 displayed no significant difference between $12 \mathrm{kPa}\left(\mathrm{R}^{2}=0.4818\right)$ and $72 \mathrm{kPa}\left(\mathrm{R}^{2}=0.6325\right)$ (Figure 2D). The estimated integrated-TF was independently calculated from VSMC area, using the trend line formula generated from isolate-1. Comparison of the measured and calculated integrated-TF revealed no significant difference between the two data sets (Figure 2E). Next, we sought to refine our model and compared the measured integrated$\mathrm{TF} /$ spreading relationship between the two independent isolates. Both isolates displayed a similar integrated-TF/spreading relationship (Figure $2 \mathrm{~F}$ ), and as there was no significant difference between the two isolates, we combined all the data and calculated the gradient of the trend line (Figure $2 \mathrm{G}$ ). This combined formula was used to calculate the integrated-TF of both isolates. Comparison revealed that the measured and calculated integrated-TF, were in close agreement with no significant difference between these data sets (Figures $2 \mathrm{H}$ and $\mathrm{I}$ ). 
A.
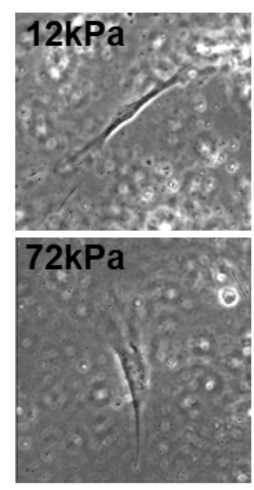

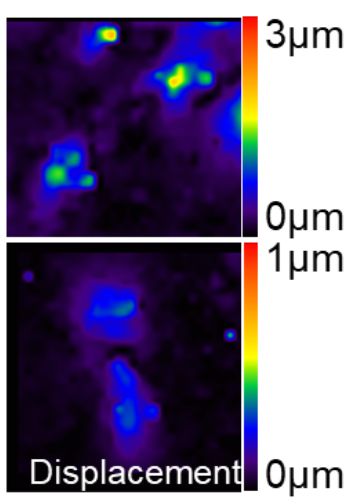

D.

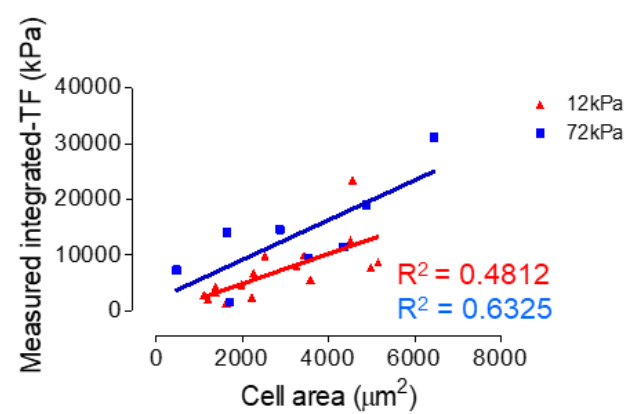

F.

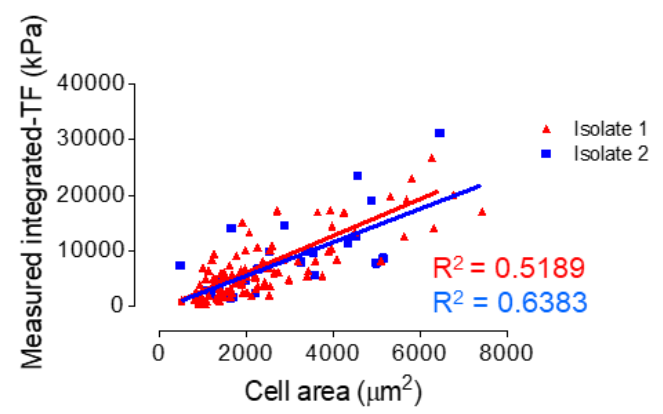

H.

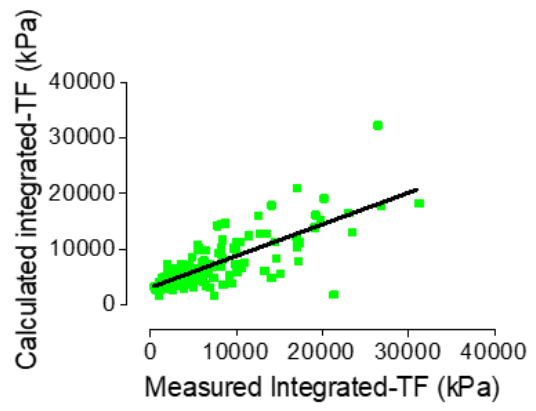

I.
B.

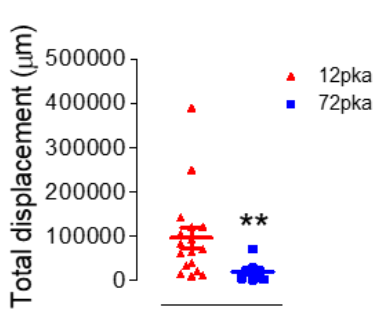

C.

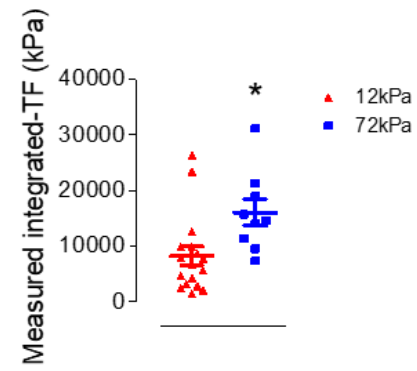

E.

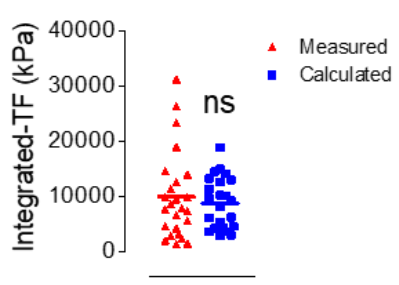

G.
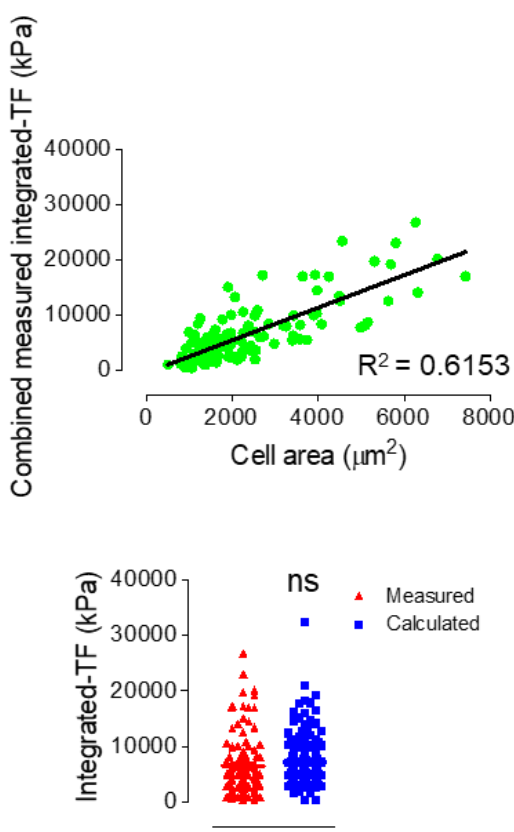

Figure 2. Refinement of the estimated integrated-TF formula. A) Representative bead displacement maps for isolate-2 VSMCs grown on 12 and $72 \mathrm{kPa}$ hydrogels. Graphs show B) integrated bead displacements and $\mathbf{C})$ integrated-TF magnitude for isolate-2 grown on $12(n=17)$ and $72 \mathrm{kPa}(\mathrm{n}=8)$ hydrogels. D) Graph shows distribution of measured integrated-TF magnitude plotted against VSMC area for isolate-2 grown on $12 \mathrm{kPa}($ red $)(n=17)$ and $72 \mathrm{kPa}$ (blue) $(n=9)$ hydrogels. E) Graph shows distribution of measured and calculated integrated-TF of isolate-2 using formula 1. F) Graph shows distribution of measured integrated-TF magnitude versus VSMC area of isolate-1 (red) $(n=104)$ and isolate-2 (blue) $(n=25)$. G) Graph shows the combined distribution of measured integrated-TF and area of isolate-1 and isolate-2 used to generate formula $2(n=129)$. H) Graph shows the combined distribution of calculated versus measured integrated-TF for isolate-1 and isolate-2. I) Graph shows the distribution of measured and calculated integrated-TF magnitude for isolate- 1 and isolate- 2 combined data. $\left({ }^{*} p=<0.05,{ }^{* *} p=\right.$ $<0.01$ and $\mathrm{ns}=$ non-significant). 
MLC phosphorylation strongly correlates with calculated integrated-TF magnitude

To further confirm that VSMC spreading and calculated integrated-TF correlated, we used an independent marker of actomyosin activity. We next performed immunofluorescence microscopy to assay phosphorylated myosin light chain (pMLC) levels in VSMCs grown on $12 \mathrm{kPa}$ and $72 \mathrm{kPa}$ hydrogels. Surprisingly, analysis revealed that pMLC mean intensity was reduced on $72 \mathrm{kPa}$ compared to $12 \mathrm{kPa}$ hydrogels, however, the total pMLC intensity remained unchanged (Figures $3 A-C$ ). magnitude strongly correlated with the total pMLC intensity on both $12 \mathrm{kPa}\left(\mathrm{R}^{2}=\right.$ $0.8658)$ and $72 \mathrm{kPa}\left(\mathrm{R}^{2}=0.9384\right)$ hydrogels (Supplementary Figure 2 and Figure 3D). However, there was no correlation between PMLC mean intensity and VSMC area/estimated integrated-TF (Supplementary Figure 2 Figure 3E). The $\mathrm{R}^{2}$ values were not significantly different between $12 \mathrm{kPa}$ and $72 \mathrm{kPa}$ hydrogels for the of matrix stiffness.

A.
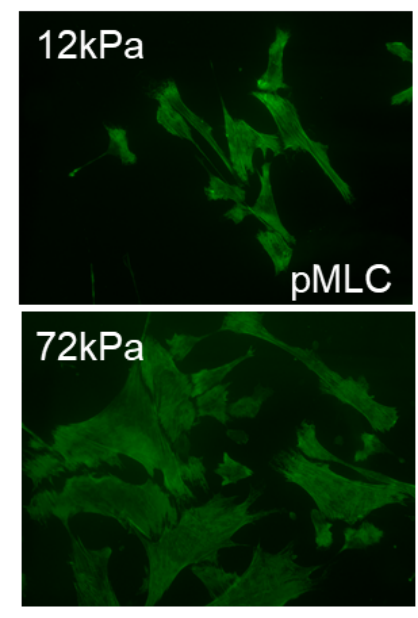

B.

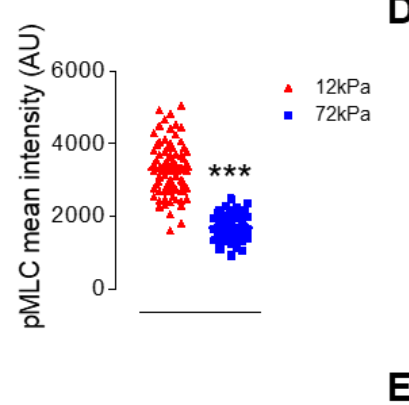

C.

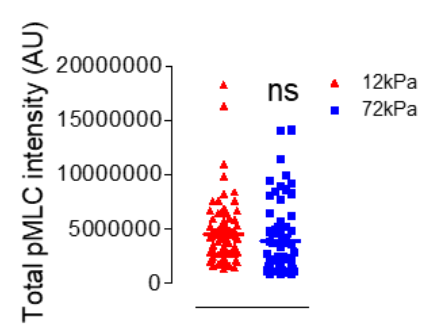

D.

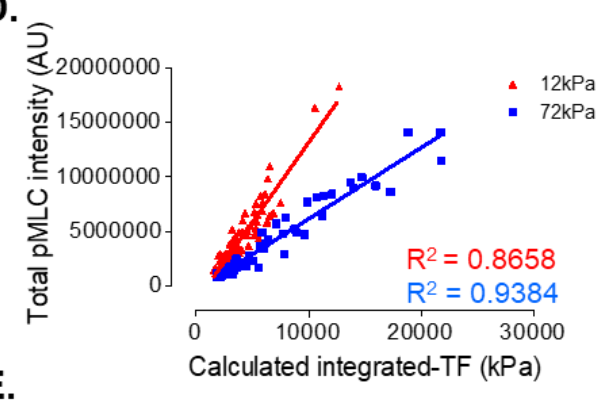

E.

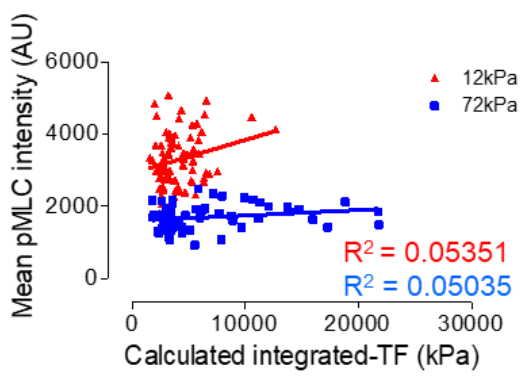

Figure 3. Total pMLC levels correlate with calculated integrated-TF. A) Representative images of pMLC stained isolate-1 VSMCs grown on 12 and $72 \mathrm{kPa}$ hydrogels. Graphs show B) pMLC mean intensity and C) pMLC total intensity in isolate- 1 VSMCs grown on $12(n=77)$ and $72 \mathrm{kPa}(n=61)$ hydrogels. Graphs show D) pMLC mean intensity and E) pMLC total intensity verses calculated integrated-TF on 12 (red) and $72 \mathrm{kPa}$ (blue) hydrogels. ( ${ }^{* * *} \mathrm{p}=<0.0001$ and $\mathrm{ns}=$ non-significant). 
Increased cell: matrix adhesion number enhances VSMC traction force

A.
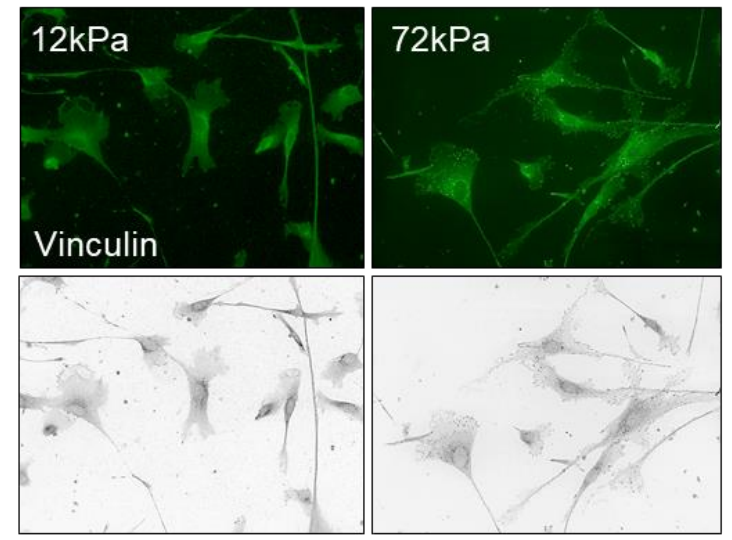

E.

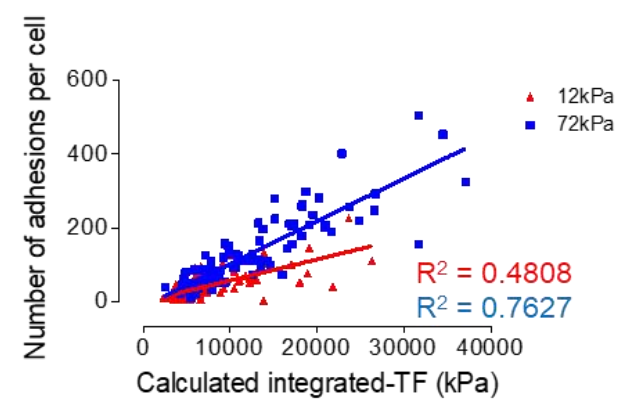

G.

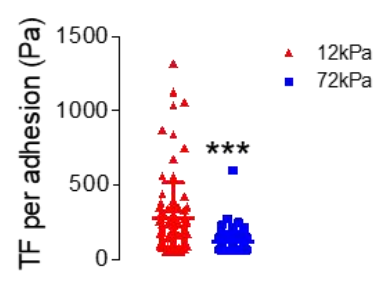

B.

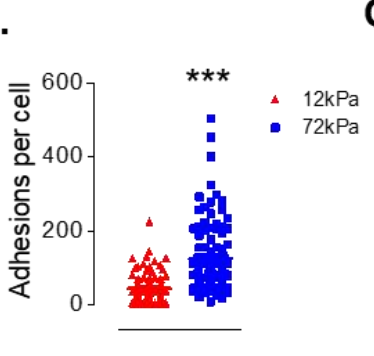

D.

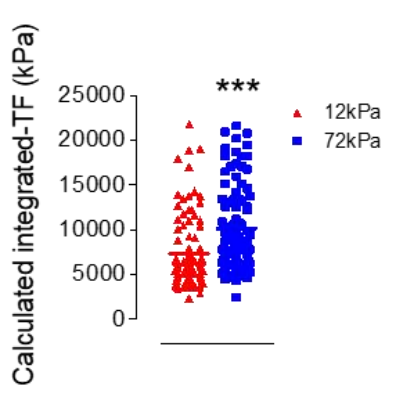

F.

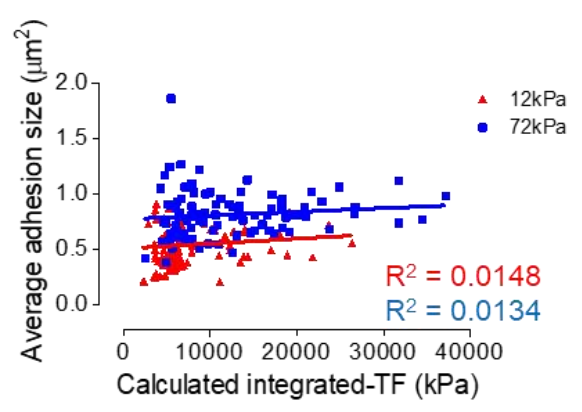

I.

Figure 4. Matrix rigidity influences VSMC matrix adhesion organisation. A) Representative images of vinculin stained isolate-1 VSMCs grown on 12 and $72 \mathrm{kPa}$ hydrogels. Graphs show B) average adhesion size and $C)$ number of adhesions per cell on $12 \mathrm{kPa}(\mathrm{n}=94)$ and $72 \mathrm{kPa}(\mathrm{n}=99)$ hydrogels. Graphs represent the combined data from 3-independent experiments. $\left.{ }^{* * *} p=<0.0001\right)$. Graphs show $D$ ) adhesion size and $E$ ) adhesion number per cell verses calculated integrated-TF on 12 (red) and $72 \mathrm{kPa}$ (blue) hydrogels. Graphs show F) calculated integrated-TF, G) integrated-TF per adhesion, $\mathrm{H}$ ) adhesion number/ $\mu \mathrm{m} 2$ and I) integrated-TF/ $\mu \mathrm{m} 2$ for VSMCs grown on 12 and $72 \mathrm{kPa}$ hydrogels. ${ }^{* \star *} p=<0.0001$ and $n s=$ non-significant). 
matrix adhesions, so we performed IF analysis of VSMCs stained with an antibody raised to the matrix-adhesion protein vinculin. Analysis revealed that VSMCs grown on $72 \mathrm{kPa}$ hydrogels possessed an increased number of matrix adhesions and larger matrix adhesions compared to VSMCs grown on $12 \mathrm{kPa}$ hydrogels (Figure $4 \mathrm{~A}-\mathrm{C}$ ). This data shows that VSMC matrix adhesions are altered by matrix rigidity. Next, using formula 2, the integrated-TF was calculated from the cell area data and confirmed that cell area and calculated integrated-TF were enhanced by matrix rigidity (Supplementary Figure 3 and Figure 4D). We next examined the relationship between VSMC spreading/calculated integrated-TF magnitude and vinculin organisation. Analysis revealed that VSMC spreading/calculated integrated-TF magnitude correlated with the number of vinculin adhesions but not adhesion size (Figures 4E, F and Supplementary Figure 3). Analysis revealed a significant difference between the $12 \mathrm{kPa}$ and $72 \mathrm{kPa}$ trend-line gradient, confirming that this relationship is enhanced by matrix rigidity (Figures $4 \mathrm{E}, \mathrm{F}$ and Supplementary Figure 3). Finally, we sought to dissect whether increased integrated-TF magnitude was driven by increased adhesion number in larger VSMCs. Analysis revealed that the average-TF per adhesion was decreased on $72 \mathrm{kPa}$ hydrogels (Figure 4G). Importantly, VSMCs grown on $72 \mathrm{kPa}$ hydrogels possessed an increased number of adhesions $/ \mu \mathrm{m}^{2}$ (Figure $4 \mathrm{H}$ ) and therefore displayed a similar TF/ $\mu \mathrm{m}^{2}$ as VSMCs on $12 \mathrm{kPa}$ hydrogels (Figure $4 \mathrm{I}$ ).

\section{Discussion}

Decreased aortic compliance is observed during vascular ageing and disease, however, our understanding of how changes in aortic stiffness influence VSMC function remains limited. Decreased aortic compliance is ultimately driven by changes in the ECM composition, including elastin fragmentation and collagen accumulation $(5,6)$. VSMCs normally adopt a quiescent, contractile phenotype in the vessel wall, however, VSMCs are not terminally differentiated and can adopt a synthetic phenotype (30). Matrix rigidity has been shown to promote VSMC proliferation, suggesting that microenvironment rigidity influences VSMC phenotype (30). Matrix rigidity is also known to influence VSMC morphology and traction force magnitude however, the mechanisms driving these processes remain to be defined $(31,32)$. In agreement with previous findings using immature embryonic aortic VSMCs, we show that VSMC traction force magnitude and spreading are enhanced by matrix rigidity. However, this 
previous study used hydrogels between $10 \mathrm{kPa}$ and $25 \mathrm{kPa}$ (32). These rigidities are within the normal range of adult aortic rigidity (4). Our study is the first to examine this phenomenon in mature adult VSMCs at a rigidity more akin to disease. In other cell types, matrix rigidity enhances the relationship between traction force magnitude and cell spreading (33). However, we show that this relationship in adult VSMCs is independent of matrix rigidity in two independent VSMC isolates. Furthermore, we have generated a model for estimating integrated-TF magnitude from VSMC spreading data. TFM is a time consuming and specialised technique that can only be performed on live cells. We have used our model to estimate integrated-TF magnitude using fixed VSMC data. Importantly, we show that in a population of smooth muscle cells there is no significant difference in between measured and calculated integratedTF.

Although increased VSMC spreading stimulated integrated-TF magnitude, $\mathrm{TF} / \mu \mathrm{m}^{2}$ remained unaltered by both matrix rigidity and spreading. This suggests that actomyosin-generated force that is transferred to the ECM by VSMCs is unaffected by spreading. However, pMLC analysis revealed that matrix rigidity reduces pMLC levels, suggesting that actomyosin activity is reduced by matrix rigidity. Matrix adhesions remodel in response to matrix rigidity and changes in both adhesion number per cell and adhesion size have been reported in a variety of cell types (34). VSMC matrix adhesion number increased with spreading, yet the average-TF per adhesion was reduced. Importantly, matrix rigidity stimulated increased matrix adhesion numbers which resulted in equivalent levels of TF/ $\mu \mathrm{m}^{2}$, as VSMCs grown on $12 \mathrm{kPa}$ hydrogels. These data suggest that enhanced VSMC anchor points and not enhanced actomyosin activity contribute to the increase in integrated-TF. Our data also shows that matrix rigidity inversely regulates pMLC levels and matrix adhesion number in VSMCs; matrix rigidity promotes decreased PMLC levels and increased adhesion anchor points. Matrix stiffness also increases VSMC spreading, however, due to this inverse $\mathrm{pMLC/adhesion} \mathrm{number} \mathrm{regulation,} \mathrm{there} \mathrm{is} \mathrm{no} \mathrm{change} \mathrm{on} \mathrm{force} \mathrm{transfer/} \mu \mathrm{m}^{2}$ between VSMCs and the ECM. As a result, the integrated-TF/VSMC spreading relationship is matrix rigidity independent. This suggests that the force/ $\mu \mathrm{m}^{2}$ exerted by VSMCs on the ECM is tightly regulated. We propose that matrix rigidity triggers a compensatory mechanism to preserve force $/ \mu m^{2}$ that potentially protects VSMCs from actomyosin-induced damage (Figure 5). Unrestrained actomyosin activity induces DNA damage in other cells types and DNA damage drives VSMC ageing (35-37). 

induced ageing.

\section{Physiological rigidity}

pMLC intensity

Actomyosin activity

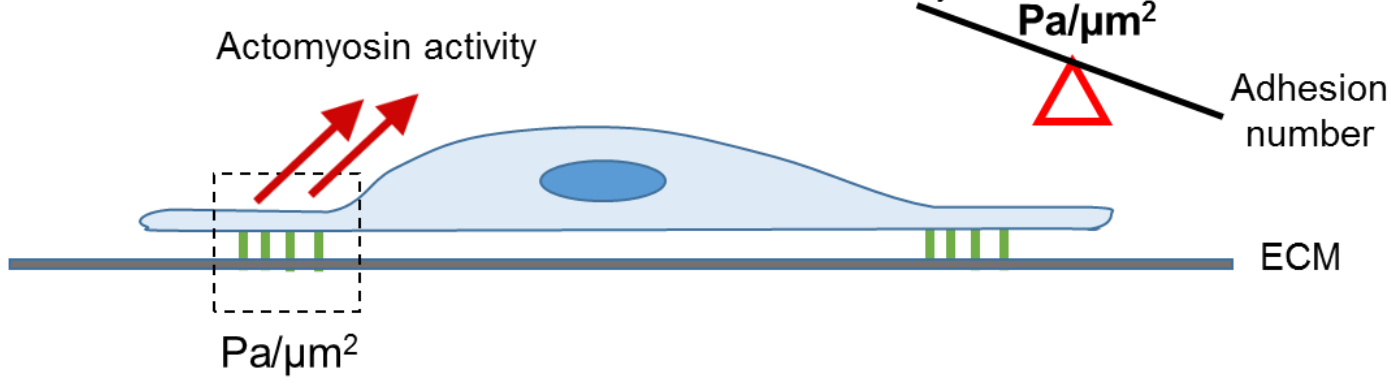

\section{Pathological rigidity}

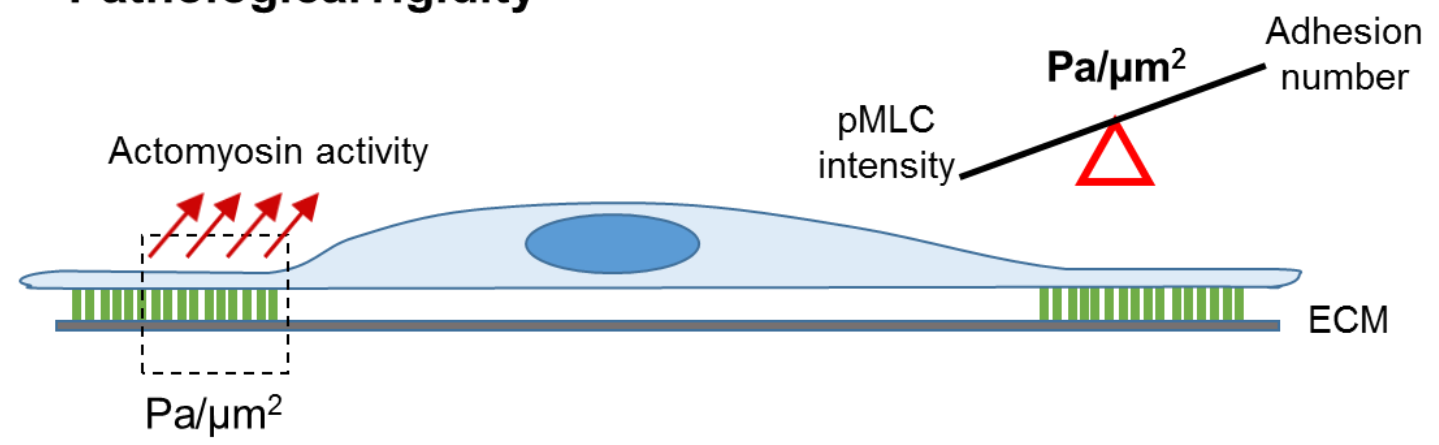

Figure 5. Model representing how differential adhesion number and actomyosin regulation preserve VSMC integrated-TF/ $\mu \mathrm{m}^{2}$.

Despite this compensatory mechanism, VSMC spreading promotes increased adhesive contractile units that ultimately increase integrated-TF. However, despite generating increased integrated-TF, the ability of VSMCs to deform the ECM diminishes with matrix rigidity. Our findings confirm that VSMCs can efficiently displace the ECM at normal $(12 \mathrm{kPa})$ aortic stiffness and this is in agreement with the role of VSMCs in the maintenance of vascular tone (7). Enhanced actomyosin activity deforms the surrounding ECM and enables VSMCs contraction resulting in decreased vascular compliance. However, our findings show that a 6 -fold increase in stiffness dramatically diminishes the ability of VSMCs to deform their microenvironment. These findings suggest that despite matrix rigidity stimulating VSMC actomyosin activity, a 6fold increase in aortic stiffness would result in the VSMCs being unable to significantly 
contribute to vascular tone and compliance. Changes in VSMC phenotype are known to influence expression of smooth muscle cell contractile proteins (38). For example, contractile VSMCs possess both smooth muscle (SM)-myosin II and non-muscle (NM)-myosin II, whereas synthetic VSMCs possess predominantly NM-myosin II (39). Although structurally similar, SM-myosin II and NM-myosin II possess different force generating capabilities/functions; NM-myosin II generates less force than SM-myosin II and NM-myosin II contributes to tonic contraction, whereas SM-myosin contribute to phasic contraction (40-42). Our understanding of the relative contribution of NMmyosin II and SM-myosin II in VSMC dysfunction remains unknown. Synthetic VSMCs were used in this study and we cannot rule out the possibility that contractile VSMCs possess a greater ability to deform stiffened matrix than their synthetic counterparts. However, given that matrix stiffness has been demonstrated to promote VSMC proliferation, the relationship between VSMC force generation, phenotype and vessel compliance is likely to be complex. As aortic stiffness and VSMC phenotype switching are observed in vascular diseases, including atherosclerosis and hypertension, further research is now needed to better understand the relationship between VSMC force generation and vessel compliance. Dramatic changes in cytoskeletal organisation and morphology are associated with VSMC phenotypic transition and our model will allow further interrogation of these pathways that influence VSMC force generation during disease associated phenotypic modulation.

\section{Funding}

This work was supported by a British Heart Foundation (BHF) Non-clinical PhD Studentship (FS/17/32/32916).

\section{Author Contributions}

SA and PM conducted experiments and performed data analysis. DTW designed the experiments and wrote the manuscript.

\section{Conflict of Interest}

Conflict of interest: none declared. 
Data availability

The raw/processed data required to reproduce these findings cannot be shared at this time due to technical or time limitations.

\section{References}

374

375

376

377

378

379

380

381

382

383

384

385

386

387

388

389

390

391

392

393

394

395

396

397

398

399

400

401

402

403

404

405

406

407

408

409

410

411

412

413

414

1. van Popele NM, Mattace-Raso FU, Vliegenthart R, Grobbee DE, Asmar R, van der Kuip DA, et al. Aortic stiffness is associated with atherosclerosis of the coronary arteries in older adults: the Rotterdam Study. J Hypertens. 2006;24(12):2371-6.

2. Gauthier-Bastien A, Ung RV, Lariviere R, Mac-Way F, Lebel M, Agharazii M. Vascular remodeling and media calcification increases arterial stiffness in chronic kidney disease. Clin Exp Hypertens. 2014;36(3):173-80.

3. Sehgel NL, Sun Z, Hong Z, Hunter WC, Hill MA, Vatner DE, et al. Augmented vascular smooth muscle cell stiffness and adhesion when hypertension is superimposed on aging. Hypertension. 2015;65(2):370-7.

4. Tracqui P, Broisat A, Toczek J, Mesnier N, Ohayon J, Riou L. Mapping elasticity moduli of atherosclerotic plaque in situ via atomic force microscopy. J Struct Biol. 2011;174(1):115-23.

5. Steed MM, Tyagi N, Sen U, Schuschke DA, Joshua IG, Tyagi SC. Functional consequences of the collagen/elastin switch in vascular remodeling in hyperhomocysteinemic wild-type, eNOS-/-, and iNOS-/- mice. Am J Physiol Lung Cell Mol Physiol. 2010;299(3):L301-11.

6. Wagenseil JE, Mecham RP. Vascular extracellular matrix and arterial mechanics. Physiol Rev. 2009;89(3):957-89.

7. Galmiche G, Labat C, Mericskay M, Aissa KA, Blanc J, Retailleau K, et al. Inactivation of serum response factor contributes to decrease vascular muscular tone and arterial stiffness in mice. Circ Res. 2013;112(7):1035-45.

8. Sehgel NL, Zhu Y, Sun Z, Trzeciakowski JP, Hong Z, Hunter WC, et al. Increased vascular smooth muscle cell stiffness: a novel mechanism for aortic stiffness in hypertension. Am J Physiol Heart Circ Physiol. 2013;305(9):H1281-7.

9. Qiu H, Zhu Y, Sun Z, Trzeciakowski JP, Gansner M, Depre C, et al. Short communication: vascular smooth muscle cell stiffness as a mechanism for increased aortic stiffness with aging. Circ Res. 2010;107(5):615-9.

10. Alexander MR, Owens GK. Epigenetic control of smooth muscle cell differentiation and phenotypic switching in vascular development and disease. Annu Rev Physiol. 2012;74:13-40.

11. Owens GK. Molecular control of vascular smooth muscle cell differentiation and phenotypic plasticity. Novartis Found Symp. 2007;283:174-91; discussion 91-3, 238-41.

12. Rzucidlo EM, Martin KA, Powell RJ. Regulation of vascular smooth muscle cell differentiation. J Vasc Surg. 2007;45 Suppl A:A25-32.

13. Chen $\mathrm{Y}$, Lee $\mathrm{H}$, Tong $\mathrm{H}$, Schwartz $\mathrm{M}$, Zhu C. Force regulated conformational change of integrin alphaVbeta3. Matrix Biol. 2017;60-61:70-85.

14. Karimi A, Milewicz DM. Structure of the Elastin-Contractile Units in the Thoracic Aorta and How Genes That Cause Thoracic Aortic Aneurysms and Dissections Disrupt This Structure. Can J Cardiol. 2016;32(1):26-34.

15. Ehrlicher AJ, Nakamura F, Hartwig JH, Weitz DA, Stossel TP. Mechanical strain in actin networks regulates FilGAP and integrin binding to filamin A. Nature. 2011;478(7368):260-3.

16. Ross TD, Coon BG, Yun S, Baeyens N, Tanaka $K$, Ouyang $M$, et al. Integrins in mechanotransduction. Curr Opin Cell Biol. 2013;25(5):613-8. 
bioRxiv preprint doi: https://doi.org/10.1101/612267; this version posted April 17, 2019. The copyright holder for this preprint (which was not certified by peer review) is the author/funder, who has granted bioRxiv a license to display the preprint in perpetuity. It is made available under aCC-BY 4.0 International license.

17. Lo $\mathrm{CM}$, Wang HB, Dembo M, Wang YL. Cell movement is guided by the rigidity of the substrate. Biophys J. 2000;79(1):144-52.

18. Friedland $\mathrm{JC}$, Lee $\mathrm{MH}$, Boettiger $\mathrm{D}$. Mechanically activated integrin switch controls alpha5beta1 function. Science. 2009;323(5914):642-4.

19. Wang YK, Wang YH, Wang CZ, Sung JM, Chiu WT, Lin SH, et al. Rigidity of collagen fibrils controls collagen gel-induced down-regulation of focal adhesion complex proteins mediated by alpha2beta1 integrin. J Biol Chem. 2003;278(24):21886-92.

20. Wei WC, Lin HH, Shen MR, Tang MJ. Mechanosensing machinery for cells under low substratum rigidity. Am J Physiol Cell Physiol. 2008;295(6):C1579-89.

21. Lampi MC, Reinhart-King CA. Targeting extracellular matrix stiffness to attenuate disease: From molecular mechanisms to clinical trials. Sci Transl Med. 2018;10(422).

22. Sehgel NL, Vatner SF, Meininger GA. "Smooth Muscle Cell Stiffness Syndrome"-Revisiting the Structural Basis of Arterial Stiffness. Front Physiol. 2015;6:335.

23. Hytonen VP, Wehrle-Haller B. Mechanosensing in cell-matrix adhesions - Converting tension into chemical signals. Exp Cell Res. 2016;343(1):35-41.

24. Warren DT, Tajsic T, Porter $L$, Minaisah RM, Cobb A, Jacob A, et al. Nesprin-2-dependent ERK1/2 compartmentalisation regulates the DNA damage response in vascular smooth muscle cell ageing. Cell Death Differ. 2015;22(9):1540-50.

25. Minaisah RM, Cox S, Warren DT. The Use of Polyacrylamide Hydrogels to Study the Effects of Matrix Stiffness on Nuclear Envelope Properties. Methods Mol Biol. 2016;1411:233-9.

26. Tse JR, Engler AJ. Preparation of hydrogel substrates with tunable mechanical properties. Curr Protoc Cell Biol. 2010; Chapter 10:Unit 106.

27. Tseng $Q$, Duchemin-Pelletier E, Deshiere A, Balland $M$, Guillou $H$, Filhol $O$, et al. Spatial organization of the extracellular matrix regulates cell-cell junction positioning. Proc Natl Acad Sci U S A. 2012;109(5):1506-11.

28. Porter L, Holt MR, Soong D, Shanahan CM, Warren DT. Prelamin A Accumulation Attenuates Rac1 Activity and Increases the Intrinsic Migrational Persistence of Aged Vascular Smooth Muscle Cells. Cells. 2016;5(4).

29. Tolic-Norrelykke IM, Wang N. Traction in smooth muscle cells varies with cell spreading. J Biomech. 2005;38(7):1405-12.

30. Shi N, Chen SY. Smooth Muscle Cell Differentiation: Model Systems, Regulatory Mechanisms, and Vascular Diseases. J Cell Physiol. 2015.

31. McDaniel DP, Shaw GA, Elliott JT, Bhadriraju K, Meuse C, Chung KH, et al. The stiffness of collagen fibrils influences vascular smooth muscle cell phenotype. Biophys J. 2007;92(5):1759-69.

32. Sazonova OV, Isenberg BC, Herrmann J, Lee KL, Purwada A, Valentine AD, et al. Extracellular matrix presentation modulates vascular smooth muscle cell mechanotransduction. Matrix Biol. 2015;41:36-43.

33. Califano JP, Reinhart-King CA. Substrate Stiffness and Cell Area Predict Cellular Traction Stresses in Single Cells and Cells in Contact. Cell Mol Bioeng. 2010;3(1):68-75.

34. Wells RG. The role of matrix stiffness in regulating cell behavior. Hepatology. 2008;47(4):1394400.

35. Herraiz C, Calvo F, Pandya P, Cantelli G, Rodriguez-Hernandez I, Orgaz JL, et al. Reactivation of p53 by a Cytoskeletal Sensor to Control the Balance Between DNA Damage and Tumor Dissemination. J Natl Cancer Inst. 2016;108(1).

36. Takaki T, Montagner M, Serres MP, Le Berre M, Russell M, Collinson L, et al. Actomyosin drives cancer cell nuclear dysmorphia and threatens genome stability. Nat Commun. 2017;8:16013.

37. Shanahan CM. Mechanisms of vascular calcification in CKD-evidence for premature ageing? Nat Rev Nephrol. 2013;9(11):661-70.

38. Shi N, Chen SY. Smooth Muscle Cell Differentiation: Model Systems, Regulatory Mechanisms, and Vascular Diseases. J Cell Physiol. 2016;231(4):777-87. 
39. Eddinger TJ, Meer DP. Myosin II isoforms in smooth muscle: heterogeneity and function. Am J Physiol Cell Physiol. 2007;293(2):C493-508.

467 40. Eddinger TJ, Meer DP, Miner AS, Meehl J, Rovner AS, Ratz PH. Potent inhibition of arterial smooth muscle tonic contractions by the selective myosin II inhibitor, blebbistatin. J Pharmacol Exp Ther. 2007;320(2):865-70. Physiol. 2003;121(4):301-10. contractile phenotype in smooth muscle. Pflugers Arch. 2006;452(6):766-74. 